\title{
GRAVITATIONAL-WAVE CONSTRAINTS ON ABUNDANCE OF PRIMORDIAL BLACK HOLES
}

\author{
RYO SAITO \\ Department of Physics, Graduate School of Science, The University of Tokyo \\ Tokyo 113-0033, Japan \\ Research Center for the Early Universe, Graduate School of Science, The University of Tokyo \\ Tokyo 113-0033, Japan \\ r-saito@resceu.s.u-tokyo.ac.jp \\ JUN'ICHI YOKOYAMA \\ Research Center for the Early Universe, Graduate School of Science, The University of Tokyo \\ Tokyo 113-0033, Japan \\ Institute for the Physics and Mathematics of the Universe, University of Tokyo \\ Chiba 277-8568, Japan \\ yokoyama@resceu.s.u-tokyo.ac.jp
}

\begin{abstract}
Formation of primordial black holes (PBHs) requires a large root-mean-square amplitude of density fluctuations, which generate second-order tensor perturbations that can be compared with observational constraints. We show that pulsar timing data essentially rules out PBHs with $10^{2-4} M_{\odot}$ which were previously considered as a candidate of intermediate-mass black hoes and that PBHs with mass range $10^{20-25} \mathrm{~g}$ which provide an astrophysical candidate for dark matter may be probed by future space-based laser interferometers.
\end{abstract}

Keywords: Primordial black hole; gravitational wave.

PACS Nos.: 04.30.Db, 04.70.-s, 98.70.Vc, 98.80.-k

\section{Introduction}

Primordial black holes (PBHs) are produced when density fluctuations with a large amplitude enters the horizon in the radiation dominated stage of the early universe with their typical mass given by the horizon mass at that epoch. ${ }^{1,2} \mathrm{PBH}$ s with their mass smaller than $10^{15} \mathrm{~g}$ would have been evaporated away by now due to the Hawking radiation and a number of cosmological constraints have been imposed on the abundance of these light holes by big-bang nucleosynthesis (BBN) and gamma-ray background etc. Heavier PBHs can play some astrophysical roles today. For example, they may serve as the origin of the intermediate-mass black holes (IMBHs), which are considered to be the observed ultra-luminous X-ray sources, if their mass and abundance lie in the range $M_{\mathrm{PBH}} \sim 10^{2} M_{\odot}-10^{4} M_{\odot}$ and $\Omega_{\mathrm{PBH}} h^{2} \sim 10^{-5}-10^{-2}$, respectively, ${ }^{3}$ while $\mathrm{PBHs}$ with mass $M_{\mathrm{PBH}} \sim 10^{20} \mathrm{~g}-10^{26} \mathrm{~g}\left(10^{-13} M_{\odot}-10^{-7} M_{\odot}\right)^{2}$ 
and the abundance $\Omega_{\mathrm{PBH}} h^{2}=0.1$ can provide astrophysical origin of dark matter (DM) which is yet free from the constraint imposed by gravitational lensing experiments. ${ }^{4}$ In order to produce the relevant density of PBHs, it is necessary to produce density fluctuations whose power spectrum has a high peak with an amplitude $10^{-2}-10^{-1}$ on the corresponding scales.

Second-order effects also generate tensor fluctuations to produce stochastic background of gravitational waves (GWs) from scalar-tensor mode coupling, ${ }^{6,7}$ whose amplitude may well exceed the first-order tensor perturbation generated by quantum effect during inflation in the current set up since the amplitude of density fluctuations required for a substantial density of PBHs is so large.

In the following sections, we show the GWs induced by scalar fluctuations as a second-order effect ${ }^{6,7}$ is a useful probe to investigate the abundance of the PBHs. We calculate spectrum of these second-order GWs in the case scalar fluctuations have a sufficiently large peak to realize formation of relevant numbers of PBHs. As a natural consequence we find that the spectrum of GWs has a peak on a scale approximately equal to the scale of the peak of the scalar fluctuations. We can therefore obtain information on the abundance of $\mathrm{PBH}$ with the horizon mass when the scale of the peak entered the Hubble radius by observing GWs with the frequency corresponding to the same comoving scale, namely, $10^{-9} \mathrm{~Hz}-10^{-8} \mathrm{~Hz}$ for IMBHs and $10^{-3} \mathrm{~Hz}-1 \mathrm{~Hz}$ for dark-matter PBHs. Fortunately, the former band is probed by the pulsar timing observations ${ }^{8,9}$ while the latter band can be observed in future by the space-based interferometers for dark matter PBHs.

\section{The Induced Gravitational Waves}

We write the perturbed metric as

$$
\mathrm{d} s^{2}=a(\eta)^{2}\left[-e^{2 \Phi} \mathrm{d} \eta^{2}+e^{-2 \Psi}\left(\delta_{i j}+h_{i j}\right) \mathrm{d} x^{i} \mathrm{~d} x^{j}\right],
$$

including both scalar perturbations, $\Phi$ and $\Psi$, and tensor perturbation, $h_{i j}$, which satisfies $\partial_{i} h_{j}^{i}=h_{i}^{i}=0$ with $h_{j}^{i} \equiv \delta^{i k} h_{k j}$. We assume the lowest-order tensor perturbation is negligible and consider that generated by the scalar mode as a second-order effect. The relevant part of the Einstein equation therefore reads

$$
h_{j}^{i^{\prime \prime}}+2 \mathcal{H} h_{j}^{i^{\prime}}-\partial^{2} h_{j}^{i}=2 \mathcal{P}_{r j}^{i s} S_{s}^{r},
$$

where a prime denotes differentiation with respect to the conformal time, $\mathcal{P}_{r j}^{i s}$ represents the projection operator to the transverse, traceless part, and $\mathcal{H} \equiv a^{\prime} / a^{6,7}$. The source term reads

$$
S_{s}^{r}=2 \partial^{r} \Psi \partial_{s} \Psi-\frac{4}{3(1+w)} \partial^{r}\left(\Psi+\mathcal{H}^{-1} \Psi^{\prime}\right) \partial_{s}\left(\Psi+\mathcal{H}^{-1} \Psi^{\prime}\right),
$$

with $w \equiv \rho / p$ being the equation-of-state parameter of the background fluid. In practice, only the radiation dominated era is relevant, so we take $w=1 / 3$ hereafter. We also neglect anisotropic stress, which is expected to give only a small correction, ${ }^{7}$ and set $\Phi=\Psi$ at linear order. In order to calculate the induced GWs up to second 
order, it is sufficient to use the linear scalar modes. Hence, we only need to solve the linear evolution equation,

$$
\Psi_{\boldsymbol{k}}^{\prime \prime}(\eta)+\frac{4}{\eta} \Psi_{\boldsymbol{k}}^{\prime}(\eta)+\frac{k^{2}}{3} \Psi_{\boldsymbol{k}}(\eta)=0
$$

for the scalar modes where $\Psi_{\boldsymbol{k}}$ represents a Fourier mode.

For our purpose we assume the following approximate form of the power spectrum of the initial fluctuations,

$$
\mathcal{P}_{\Psi}(k) \equiv \frac{k^{3}}{2 \pi^{2}}\left\langle\left|\Psi_{\boldsymbol{k}}(0)\right|^{2}\right\rangle=\mathcal{A}^{2} \delta\left(\ln \left(k / k_{p}\right)\right),
$$

where $k_{p}$ and $\mathcal{A}^{2}$ represent the wavenumber of the peak and (amplitude) ${ }^{2} \times$ $\ln$ (peak width) of the original spectrum, respectively. With this power spectrum the fractional energy density of the region collapsing into $\mathrm{PBH}$ s at their formation time is estimated as

$$
\beta\left(M_{\mathrm{PBH}}\right) \sim 0.1 \exp \left(-\frac{\Psi_{c}^{2}}{2 \mathcal{A}^{2}}\right),
$$

where $M_{\mathrm{PBH}}$ is of the order of the horizon mass when the comoving scale $k_{p}^{-1}$ enters the Hubble radius and $\Psi_{c}$ is the threshold value of $\mathrm{PBH}$ formation. Carr ${ }^{5}$ takes the threshold value of the density contrast to be $\delta_{c}=1 / 3$ corresponding to $\Psi_{c}=1 / 2$. One can express the current value of the density parameter of $\mathrm{PBHs}$ in terms of $\beta\left(M_{\mathrm{PBH}}\right)$ as

$$
\Omega_{\mathrm{PBH}, 0} h^{2}=2 \times 10^{6} \beta\left(M_{\mathrm{PBH}}\right)\left(\frac{M_{\mathrm{PBH}}}{10^{36} \mathrm{~g}}\right)^{-1 / 2}\left(\frac{g_{* p}}{10.75}\right)^{-1 / 3},
$$

where $g_{* p}$ is the effective number of the relativistic degrees of freedom when $k_{p}$ entered the Hubble radius.

We define the Fourier modes $h_{\boldsymbol{k}}$ as

$$
h_{i j}(\boldsymbol{x}, \eta)=\int \frac{\mathrm{d}^{3} k}{(2 \pi)^{3 / 2}} e^{i \boldsymbol{k} \cdot \boldsymbol{x}}\left[h_{\boldsymbol{k}}^{+}(\eta) \mathrm{e}_{i j}^{+}(\boldsymbol{k})+h_{\boldsymbol{k}}^{\times}(\eta) \mathrm{e}_{i j}^{\times}(\boldsymbol{k})\right],
$$

where $\mathrm{e}_{i j}^{+}(\boldsymbol{k}), \mathrm{e}_{i j}^{\times}(\boldsymbol{k}) \quad$ are polarization tensors which are normalized as $\sum_{i, j} \mathrm{e}_{i j}^{\alpha}(\boldsymbol{k}) \mathrm{e}_{i j}^{\beta}(-\boldsymbol{k})=2 \delta^{\alpha \beta}$. The Fourier transform of the source term (3) is also defined similarly. We find the source term is constant when $k_{p} \eta / \sqrt{3} \ll 1$, while it decreases in proportion to $\eta^{-2}$ for $k_{p} \eta / \sqrt{3} \gg 1$. As a result the production of scalar-induced GWs mostly occurs around the time when $k_{p}$ crosses the sound horizon. In the mass range of the PBHs of our interest, creation of scalar-induced GWs is terminated well before the equality time. After that the energy density of GWs decreases in proportion to $a^{-4}$. Using the Green function method one can easily find a formal solution to (2) from which we can evaluate the density parameter of GWs contributed by a logarithmic interval of the wavenumber around $k$. It is formally expressed as

$$
\Omega_{\mathrm{GW}}(k, \eta)=\frac{k^{3}}{12 \pi^{2} \mathcal{H}^{2}}\left(\left|h_{\boldsymbol{k}}^{+^{\prime}}\right|^{2}+\left|h_{\boldsymbol{k}}^{\times^{\prime}}\right|^{2}\right)
$$


when $k$-mode is well inside the horizon. The overall feature of the spectrum of $\Omega_{\mathrm{GW}}\left(f, \eta_{0}\right)$ today can be estimated to be

$$
\begin{aligned}
\Omega_{\mathrm{GW}}(f) h^{2} & =7 \times 10^{-9}\left(\frac{g_{* p}}{10.75}\right)^{-1 / 3}\left(\frac{\mathcal{A}^{2}}{10^{-3}}\right)^{2} \\
& \times\left(\frac{f}{f_{p}}\right)^{2}\left[1-\left(\frac{f}{2 f_{p}}\right)^{2}\right]^{2} \theta\left(1-\frac{f}{2 f_{p}}\right) \\
& \equiv A_{\mathrm{GW}}\left(\frac{f}{f_{p}}\right)^{2}\left[1-\left(\frac{f}{2 f_{p}}\right)^{2}\right]^{2} \theta\left(1-\frac{f}{2 f_{p}}\right),
\end{aligned}
$$

for $f \lesssim f_{\mathrm{GW}} \equiv 2 f_{p} / \sqrt{3}$ where we have used the frequency $f \equiv 2 \pi k / a_{0}$ instead of the wavenumber. The above expression has the peak value $\Omega_{\mathrm{GW}}\left(f_{\mathrm{GW}}\right) h^{2}=$ $(16 / 27) A_{\mathrm{GW}}$ at $f_{\mathrm{GW}}$.

Note, however, that the actual spectrum of GW calculated from (5) has a much larger and sharper peak at $f_{\mathrm{GW}}$ besides the bulk spectrum (10) due to the resonant amplification between the transfer function and the Green function. ${ }^{6}$ Such an amplification occurs if the peak width, $\Delta$, of the primordial scalar fluctuation is sufficiently small, $\Delta \ll k_{p} / 2$. Since the resonant growth of the amplitude depends on the detailed shape of the spectrum around the peak, we do not incorporate it to yield a conservative bound on the $\mathrm{PBH}$ abundance independent of it.

\section{Constraints on the PBH Abundance}

We now compare our results with observational constraints. For definiteness we identify $M_{\mathrm{PBH}}$ with the horizon mass when the peak scale $k_{p}^{-1}$ entered the Hubble radius. Then $M_{\mathrm{PBH}}$ is related with the peak frequency of GWs as

$$
f_{\mathrm{GW}}=1 \times 10^{-8} \mathrm{~Hz}\left(\frac{M_{\mathrm{PBH}}}{10^{36} \mathrm{~g}}\right)^{-1 / 2}\left(\frac{g_{* p}}{10.75}\right)^{-1 / 12} .
$$

The pulsar timing observations are sensitive to GWs with $f>1 / T$ where $T$ is the data span. The 7-year data of observation of PSR B1855+09 gives an upper limit

$$
\Omega_{\mathrm{GW}}(f) h^{2}<4.8 \times 10^{-9}\left(\frac{f}{4.4 \times 10^{-9} \mathrm{~Hz}}\right)^{2},
$$

for $f>4.4 \times 10^{-9} \mathrm{~Hz}$ at $90 \%$ confidence level. ${ }^{8}$ By using this limit, we can constrain the abundance of PBHs with mass $M_{\mathrm{PBH}} \lesssim 1 \times 10^{37} \mathrm{~g}=5 \times 10^{3} M_{\odot}$.

The space-based interferometers are sensitive to GWs with $10^{-5} \mathrm{~Hz} \lesssim f \lesssim 10 \mathrm{~Hz}$, which covers the entire mass range of the PBHs which are allowed to be DM, $10^{20} \mathrm{~g}<M_{\mathrm{PBH}}<10^{26} \mathrm{~g}$. LISA will have its best sensitivity $\Omega_{\mathrm{GW}} h^{2} \sim 10^{-11}$ at $f \sim 10^{-2} \mathrm{~Hz}\left(M_{\mathrm{PBH}} \sim 10^{24} \mathrm{~g}\right), \mathrm{BBO}$ and the ultimate-DECIGO are planned to have sensitivity to $\Omega_{\mathrm{GW}} h^{2} \sim 10^{-13}$ and $\Omega_{\mathrm{GW}} h^{2} \sim 10^{-17}$, respectively at $f \sim$ $10^{-1} \mathrm{~Hz}\left(M_{\mathrm{PBH}} \sim 10^{22} \mathrm{~g}\right)$. 


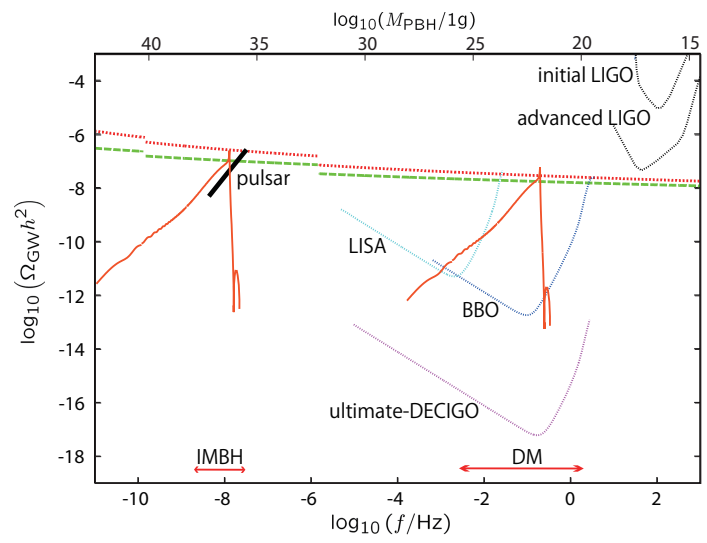

Fig. 1. (Color online) Energy density of scalar-induced GWs associated with PBH formation together with current pulsar constraint (thick solid line segment) and sensitivity of various GW detectors (convex curves). Left and right wedge-shaped curves indicate expected power spectra of GWs from two different peaked scalar fluctuations corresponding to $\left(\Omega_{\mathrm{PBH}} h^{2}, M_{\mathrm{PBH}}, g_{* p}\right)=$ $\left(10^{-5}, 6 \times 10^{2} M_{\odot}, 10.75\right)$ (left) and $\left(10^{-1}, 3 \times 10^{22} \mathrm{~g}, 106.75\right)$ (right), respectively. The red dotted (green broken) line shows an envelope curve, $(16 / 27) A_{\mathrm{GW}}$, corresponding to $\Omega_{\mathrm{PBH}}=10^{-1}\left(10^{-5}\right)$ obtained by moving $k_{p}$ and $\mathcal{A}$.

Figure 1 shows the energy density of the induced GWs, whose approximate form is given by (10). The left wedge-shaped curve represents the case $k_{p}=0.2 \mathrm{pc}^{-1}$ and $\mathcal{A}=7 \times 10^{-2}$ corresponding to $M_{\mathrm{PBH}}=6 \times 10^{2} M_{\odot}$ and $\Omega_{\mathrm{PBH}} h^{2}=10^{-5}$, while the right wedge-shaped curve depicts the case $k_{p}=1 \times 10^{6} \mathrm{pc}^{-1}$ and $\mathcal{A}=9 \times 10^{-2}$ corresponding to $M_{\mathrm{PBH}}=3 \times 10^{22} \mathrm{~g}$ and $\Omega_{\mathrm{PBH}} h^{2}=10^{-1}$. Also shown there are two envelope curves of the peak value, $(16 / 27) A_{\mathrm{GW}}$, corresponding to $\Omega_{\mathrm{PBH}} h^{2}=$ $10^{-1}$ (red dotted line) and $\Omega_{\mathrm{PBH}} h^{2}=10^{-5}$ (green broken line) which depend on the frequency logarithmically except for the discontinuities due to the change of the relativistic degrees of freedom at the QCD phase transition and the electronpositron pair annihilation. We have also shown the limit imposed by the pulsar timing observation (thick solid line segment) and the planned sensitivity of the space-based interferometers depicted ${ }^{10}$ with the instrumental parameters used in Ref. 11 as well as those of LIGO. ${ }^{12}$

As is seen in the figure the pulsar timing constraint is so stringent that one cannot achieve $\Omega_{\mathrm{PBH}} h^{2} \geq 10^{-5}$ for PBHs with $4 \times 10^{2} M_{\odot} \lesssim M_{\mathrm{PBH}} \lesssim 5 \times 10^{3} M_{\odot}$, ruling out the major mass range of IMBHs. On the other hand, if pulsar timing experiments should find any nontrivial modulation in near future, it might be due to the PBHs with mass around $10^{2} M_{\odot} \cdot{ }^{9}$

It is clear from Fig. 1 that the future space-based interferometers can test the feasibility of PBHs as the dominant constituent of the DM. The ground-based interferometers, on the other hand, have good sensitivity at $f \sim 10-10^{2} \mathrm{~Hz} .{ }^{12}$ This frequency band corresponds to mass scale $M_{\mathrm{PBH}} \sim 10^{16} \mathrm{~g}-10^{18} \mathrm{~g}$. Though the sensitivity of LIGO is too low now and in near future to detect GWs from the 
second-order effect associated with $\mathrm{PBH}$ formation, we could improve the sensitivity by correlation analysis to reach the desired level to probe PBHs. Because the spectrum has a tail to lower frequencies, it may be possible to constrain the abundance of the PBHs with $M_{\mathrm{PBH}}<7 \times 10^{14} \mathrm{~g}\left(f_{\mathrm{GW}}>6 \times 10^{2} \mathrm{~Hz}\right)$, which have evaporated by the present epoch and could contribute to cosmic rays. Further study, however, is necessary in order to obtain the conclusion because there are astronomical sources of GWs in this frequency band, too.

In summary, we have calculated the spectrum of the stochastic gravitational wave background generated as a second-order effect from scalar perturbations which have a spectrum with a high peak to realize formation of appreciable numbers of PBHs. As a result we have found that PBHs with their mass corresponding to that of IMBHs are already being ruled out because the amplitude of the associated GWs exceeds the limit imposed by the pulsar timing. We have also found that if $\mathrm{PBHs}$ with mass $10^{20}-10^{26} \mathrm{~g}$ are dominant constituents of DM, we can easily detect the relevant GWs by future space-based laser interferometers. Thus gravitational waves are new and powerful probe the mass spectrum of PBHs.

\section{References}

1. S. Hawking, Mon. Not. Roy. Astron. Soc. 152, 75 (1971); B. J. Carr and S. W. Hawking, Mon. Not. Roy. Astron. Soc. 168, 399 (1974).

2. B. J. Carr, In Proc. 59th Yamada Conference "Inflating Horizons of Particle Astrophysics and Cosmology", (Universal Academy Press, Tokyo, 2005) p 129.

3. T. Kawaguchi, M. Kawasaki, T. Takayama, M. Yamaguchi and J. Yokoyama, Mon. Not. Roy. Astron. Soc. 388, 1426 (2008).

4. C. Alcock et al. [MACHO Collaboration and EROS Collaboration], Astrophys. J. Lett. 499, L9 (1998); P. Tisserand et al. [EROS-2 Collaboration], Astron. Astrophys. 469, 387 (2007); G. F. Marani, R. J. Nemiroff, J. P. Norris, K. Hurley and J. T. Bonnell, Astrophys. J. Lett. 512, L13 (1999).

5. B. J. Carr, Astrophys. J. 201, 1 (1975).

6. K. N. Ananda, C. Clarkson and D. Wands, Phys. Rev. D 75, 123518 (2007).

7. D. Baumann, P. J. Steinhardt, K. Takahashi and K. Ichiki, Phys. Rev. D 76, 084019 (2007).

8. S. E. Thorsett and R. J. Dewey, Phys. Rev. D 53, 3468 (1996).

9. F. A. Jenet et al., Astrophys. J. 653, 1571 (2006).

10. http://www.srl.caltech.edu/ shane/sensitivity/

11. H. Kudoh, A. Taruya, T. Hiramatsu and Y. Himemoto, Phys. Rev. D 73, 064006 (2006).

12. http://www.ligo.caltech.edu/ 